\title{
Predicting deceased donor kidney transplant outcomes: Comparing KDRI/KDPI with machine learning
}

\section{CURRENT STATUS: POSTED}

Research Square

Eric Pahl

University of lowa

च eric-pahl@uiowa.eduCorresponding Author

ORCiD: https://orcid.org/0000-0002-8635-3012

Nick Street

University of lowa

Hans Johnson

University of lowa

Alan Reed

University of lowa

DOI:

$10.21203 / \mathrm{rs} .2 .16892 / \mathrm{v} 2$

SUBJECT AREAS

Medical Informatics

KEYWORDS

Kidney transplantation, end-stage renal disease, KDRI/KDPI, machine learning 


\section{Abstract}

The authors have withdrawn this preprint from Research Square 